\section{Oral surgery: part 1. Introduction and the management of the medically compromised patient}

\author{
T. Renton, ${ }^{* 1}$ S. Woolcombe, ${ }^{2}$ T. Taylor ${ }^{2}$ and C. M. Hill ${ }^{3}$
}

VERIFIABLE CPD PAPER
IN BRIEF

- Highlights that there remain multiple indications for oral surgery and the subject interfaces with many other dental and medical specialties.

- Stresses recognition of the medically compromised patient is paramount in order to deliver successful treatment.

- Suggests simple strategies highlighted in this chapter will prevent complications related to medical problems.

This paper is intended to provide an overview of current thinking in the more relevant medical conditions to oral surgery for primary care practitioners, giving the detail necessary to assist the primary dental clinical team in caring for patients with oral complaints that may be seen in general dental practice. Space precludes inclusion of discussion and illustration of uncommon or more complex medical conditions. Clinical tips for planning, execution and post-surgical care are discussed, and a brief overview of pre-operative, peri- and post-operative care is provided to minimise risk.

\section{INTRODUCTION}

Oral surgery is principally provided in the primary care setting, with about three million exodontia-related claims submitted to the general dental services (GDS) in the UK annually. Studies comparing the effectiveness and efficiency of third molar surgery have shown that treatment outcomes are comparable between hospital and practice. Whilst the number of cases of routine exodontia vastly exceeds the number of surgical procedures performed, figures from the GDS show that the pattern of dentoalveolar surgery carried out in general dental practice is changing. There are distinct advantages to all in the provision of oral surgery in the primary care setting

\begin{tabular}{l} 
ORAL SURGERY* \\
\hline Part 1.
\end{tabular}

'Professor Oral Surgery, King's College London; ${ }^{2}$ Senior Specialty Doctor in Oral Surgery, King's College London; ${ }^{3}$ Honorary Senior Lecturer at Cardiff University, Cardiff Dental Hospital

${ }^{*}$ Correspondence to: Professor Tara Renton

Email: tara.renton@kcl.ac.uk

Accepted 16 July 2013

DOI: $10.1038 /$ sj.bdj.2013.830

${ }^{\circledR}$ British Dental Journal 2013; 215: 213-223 including convenience for the patients, tailored care and cost-effectiveness for the taxpayer. Thus, dental practitioners have an essential role in providing oral surgery care for their patients alongside trained specialists in the primary care setting.

Since the abolition of the Dental Practice Board in 2000, figures indicating the number of wisdom teeth extracted, surgicallyremoved teeth and apicectomies performed over the last ten years are difficult to establish, as banding for the remuneration for procedures is now non-specific in the dental contract. The number of wisdom teeth extractions in secondary care has significantly reduced since the introduction of the Royal College of Surgeons England (RCS) guidelines in 1997 and National Institute of Clinical Excellence (NICE) guidelines in 2000; however, the age of patients having treatment is rising, thus increasing the complexity of surgery and the associated risks.

An enhanced level of expertise and increased confidence of dental practitioners to provide routine care and to specialise in the primary care setting should mirror those recent changes seen in medicine. However, despite this drive towards treatment in the primary care setting, research has shown that dental practitioners are becoming increasingly reluctant to undertake more complex surgery in the primary care setting, resulting in significant increases in referrals to secondary care.
This may be due to several factors including:

- Reduced exposure to oral surgery during undergraduate years

- Lack of exposure to procedures during foundation training

- Insufficient provision of specialist training.

The major hurdle to the provision of primary care oral surgery is the lack of specialists and poor remuneration for often complex and high risk procedures.

\section{The scope of oral surgery in the general dental service}

Oral surgery interfaces with many other disciplines, including orthodontics and restorative dentistry, and now plays an increasingly important role in the overall treatment of patients. The recent oral surgery review by Medical Education England ${ }^{1}$ highlights the need to develop training and specialisation in oral surgery in both primary and secondary care settings. Thus the scope for the provision of surgical treatment in the GDS is broadening. There is now an increase in the numbers of specialist practices operating as dentists with special interests (DwSIs), some with membership diplomas in surgical dentistry. The membership in oral surgery is to be established by the Royal Surgical Colleges, acting as an exit examination after a three-year core competency 
training programme. Oral surgery training programmes are being established all over the UK, a trend that will continue in reflection of workforce needs assessment. The programmes are being developed in parallel with other surgical specialty training, with competency work-based training assessments using the Intercollegiate Surgical Curriculum Programme (ISCP). The development of the Intercollegiate Fellowship in Oral Surgery (IFSE) examination, providing end fellowships for postCCST (Certificate of Completion of Surgical Training) in oral surgery, leads to eligibility for consultant post appointments.

Whilst the current General Dental Council regulations propose that new graduates should be able to perform simple exodontia and transalveolar procedures only, a thorough grounding in dentoalveolar surgery is clearly a useful weapon in the armamentarium of the general practitioner. Additionally, up-to-date theory and practical techniques will improve not only the practitioner's confidence, but also result in greater enjoyment of practice. This series is intended to provide students and practitioners - in a hospital or practice setting - with an evidence-based guide to the planning and execution of oral surgery. The content, encompassing the full range of oral surgery practised in the GDS today, includes basic routine techniques as well as surgical orthodontics, soft tissue lesions and dental trauma, but excludes implantology, which is the subject of a separate $B D J$ clinical guide.

\section{TREATMENT PLANNING FOR ORAL SURGERY IN GENERAL PRACTICE}

No matter what specialty practice one resides in, the principal responsibility of the clinician is to the patient as a whole. This holistic approach should always be applied when consulting one's patients. A patient will often confide in health problems to their dentist during assessment and it is the dentist's responsibility to provide general health advice and more specific guidance where necessary. Successful treatment, both in terms of surgical outcome and financial gain, depends on sound treatment planning (Fig. 1). The cornerstones of treatment planning are:

- Case selection and communication

- Appropriate training of your team

- Preparation for surgery

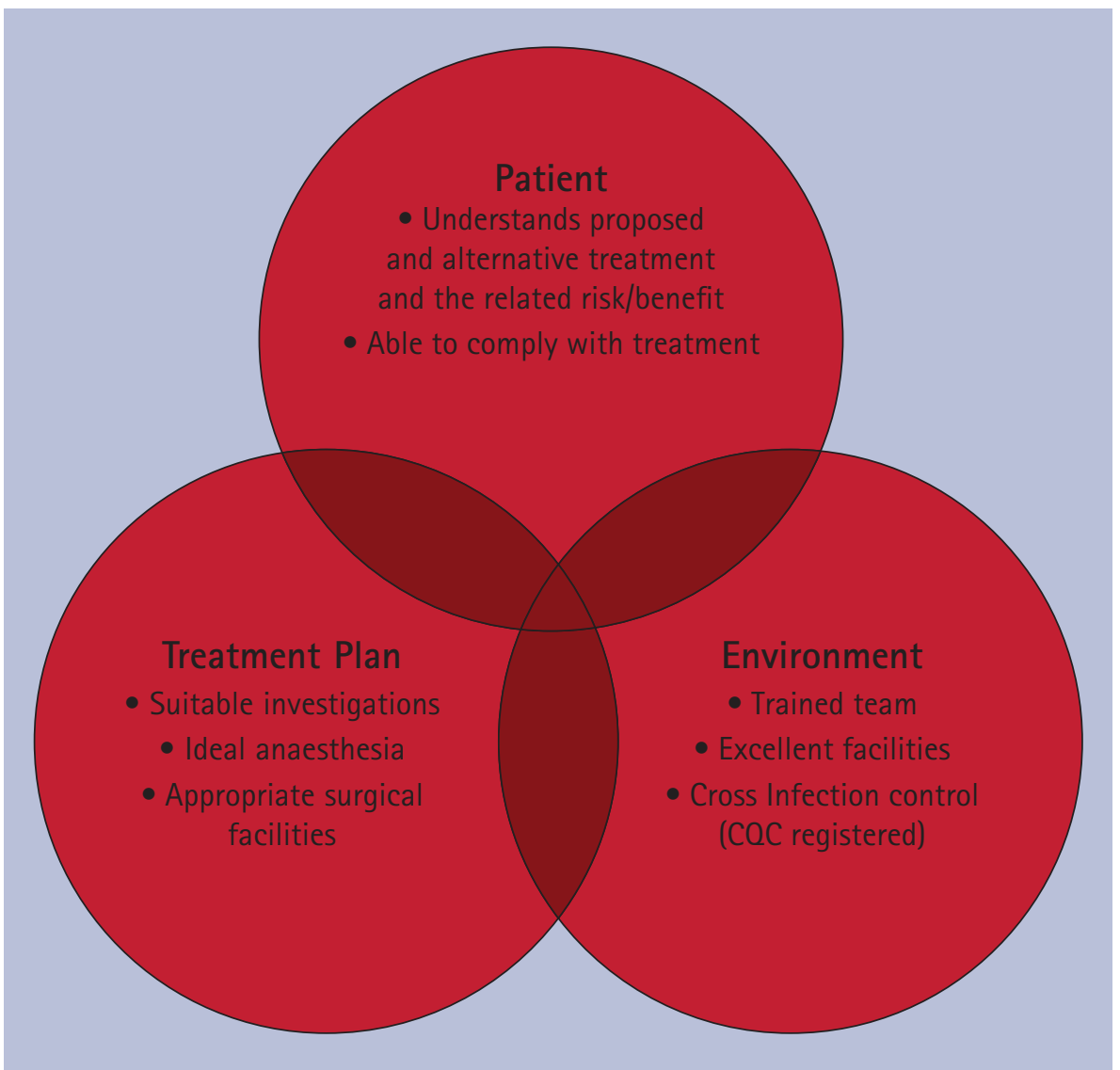

Fig. 1 Factors to consider in appropriate treatment planning and execution

- Operative technique

- Appropriate rehabilitation.

While countless studies have shown that operative skills will improve with practice, poor case selection and inadequate treatment planning can result in complications far beyond those related to the surgery alone.

\section{Patient and case selection}

Clinical assessment should be carried out with the aim of assessing the status of the patient and their surgical requirements, excluding other causes of the symptoms. Initial assessment should include:

- Patient complaint

- Patient age

- Dental history

- Social history

- A full medical history

- Extra-oral clinical examination

- Intra-oral clinical examination.

Positive findings from this examination, which suggest that treatment of the teeth or related structures may be indicated, may require that a more detailed examination is carried out. This should determine whether removal of a tooth or surgery is indicated and/or advisable and should include radiological assessment. Essentially the patient must have good compliance, good mouth opening and a normal gag reflex; any deficiency of these factors will significantly complicate routine dental extractions.

The principal decisions to be made will relate to the appropriate setting for treatment, the mode of anaesthesia and whether individual patients should be treated on an in- or out-patient basis. While the selection of mode and administration of anaesthesia is comprehensively dealt with in a later chapter, a structured outline to the factors involved in planning surgery is valuable. These relate primarily to the individual patient but the surgeon and the practice environment all have an important bearing on treatment planning.

\section{DENTAL HISTORY}

The past dental history of patients must be considered before embarking on any surgical procedures. It is clearly important to record any intra- or post-operative complications such as difficult extractions, haemorrhage or anaesthetic difficulties that have occurred previously. It 


\section{Table 1 Management of the medically compromised patient and recent controversies}

\begin{tabular}{|c|c|c|}
\hline Medical condition & Recommendations & Reference \\
\hline \multicolumn{3}{|l|}{ Cardiovascular problems: } \\
\hline $\begin{array}{l}\text { Hypertension } \\
\text { - Bleeding } \\
\text { - Risk of MI and stroke }\end{array}$ & $\begin{array}{l}\text { Hypertension } \\
\text { up to } 160 / 100 \mathrm{mmHg} \\
\text { - Treat as normal } \\
>160 / 100 \mathrm{mmHg} \\
\text { - Haemostatic agent postop } \\
\text { - IV sedation is preferable }\end{array}$ & $2,3,4$ \\
\hline $\begin{array}{l}\text { Angina } \\
\text { - Angina attack } \\
\text { - Risk of Ml }\end{array}$ & $\begin{array}{l}\text { Angina } \\
\bullet \text { Ensure glyceryl trinitrate spray and oxygen available }\end{array}$ & \\
\hline Recent MI & $\begin{array}{l}\text { MI } \\
3 \text { months post } \\
\text { - No elective treatment } \\
\text { Up to } 6 \text { months } \\
\text { - No general anaesthetic, 50\% increased risk of repeat MI }\end{array}$ & \\
\hline $\begin{array}{l}\text { Cardiac defects/valve replacements/previous endocarditis/ } \\
\text { hypertrophic cardiomyopathy: } \\
\text { Antibiotic cover for dental treatment not required. }\end{array}$ & $\begin{array}{l}\text { - No antibiotic cover } \\
\text { - Maintain good oral hygiene } \\
\text { - Warn re infective endocarditis } \\
\text { - Warn patients of symptoms of infective endocarditis - progressive malaise, } \\
\text { fever, pallor, fatigue, Janeway lesions on palms and soles of feet, splinter haem- } \\
\text { orrhages, Osler's nodes of the distal fingers }\end{array}$ & 5 \\
\hline $\begin{array}{l}\text { Liver disease: } \\
\text { - Bleeding problems } \\
\text { - Impaired drug metabolism } \\
\text { - Cross infection risk Hep B,C,D,E } \\
\text { - May be immunocompromised }\end{array}$ & $\begin{array}{l}\text { Pre-op } \\
\text { - Liaise with physician } \\
\text { - Liver profile, coagulation screen, FBC } \\
\text { - Caution with administration of LA and sedation } \\
\text { - Drug prescription check BNF appendix } 2 \text { on liver disease } \\
\text { Postop } \\
\text { - Haemostatic agent in socket } \\
\text { - Hep B immunity, caution with Hep C patients cross-infection measures in place }\end{array}$ & \\
\hline $\begin{array}{l}\text { Kidney disease: } \\
\text { - Bleeding tendency } \\
\text { - Drug prescription } \\
\text { - Dialysis patients } \\
\text { - May be immunocompromised }\end{array}$ & $\begin{array}{l}\text { Pre-op } \\
\text { - Liaise with physician } \\
\text { - Renal profile, FBC } \\
\text { - Dialysed patients to be treated the day after dialysis } \\
\text { - May require antibiotic cover } \\
\text { Postop } \\
\text { - Haemostatic measures }\end{array}$ & \\
\hline $\begin{array}{l}\text { Diabetes: } \\
\text { - Hypoglycaemic emergency } \\
\text { - Delayed healing and immunocompromised } \\
\text { - HbA1c prior to implant placement }\end{array}$ & $\begin{array}{l}\text { Pre-op } \\
\text { - Measure blood glucose level } \\
<5.0 \mathrm{mmol} \text { - administer glucose orally } \\
\text { - Morning appointment } \\
\text { - Patients safe to treat if blood sugar between } 5-15 \mathrm{mmol} / \mathrm{L} \\
\text { Postop } \\
\text { - Antibiotics if poorly controlled or difficult surgical procedure }\end{array}$ & 6 \\
\hline $\begin{array}{l}\text { Epilepsy: } \\
\text { - Increased stress may cause seizure }\end{array}$ & $\begin{array}{l}\text { - Check frequency and presentation of seizures } \\
\text { - IV sedation recommended due to anticonvulsant effects }\end{array}$ & \\
\hline
\end{tabular}

is essential that the patient has a realistic expectation of the duration, extent and complications associated with a particular surgical procedure. To this end, standard information sheets for common surgical procedures are invaluable. Insufficient explanation of both the complications and outcomes of surgery are frequent causes of legal action following dentoalveolar procedures, even after routine 'problems'.

\section{SOCIAL HISTORY}

The patient's social circumstances are an important and often overlooked element of the history in planning patient care. While oral surgery is often erroneously described as 'minor' oral surgery it should be borne in mind that patients will often experience considerable postoperative pain and interference with their normal daily routine. Moreover, patients who are either immobile or who live alone experience practical difficulties in receiving postoperative care and treatment for any complications which may arise. The number of such patients seen in practice is likely to increase as the mean age of the population increases. Social factors are important and it must be remembered that patients in socioeconomic groups who have no support in the 'community', may have to be treated on an in-patient basis. If 


\section{Table 1 Management of the medically compromised patient and recent controversies}

\begin{tabular}{|l|l|l}
\hline Medical condition & Recommendations & Reference
\end{tabular}

\section{Continued from page 215}

\section{Disorders of haemostasis:}

- Increased risk of bleeding postop
Haemophilia A, B, Von Willebrand's

- Liaise with haematology physician/haemophilia centre

- Factor VIII levels between 50-75\% required prior to treatment

- DDAVP, tranexamic acid may be needed

- Treat in hospital may require in-patient management

- Avoid inferior dental blocks if possible

Thrombocytopenia

Pre-op

- Liaise with haematology physician

- Platelet levels $>50 \times 109 / \mathrm{L}$ OK to treat, advisable to treat in hospital setting

- $<50 \times 109 / \mathrm{L}$ will require platelet transfusion

Postop

- Local haemostatic measures

- Platelets may be needed

- DDAVP, tranexamic acid

- No NSAIDs

\section{Anticoagulant therapy:}

- Increased risk of bleeding for patients on warfarin, heparin, aspirin, clopidogrel, dipyridamole, glycoprotein Ilb/IIla inhibitors, new oral anticoagulants dabigatran, apixaban and rivaroxaban (do not increase INR)

- Do not stop anticoagulant therapy; increased risk of thromboembolic event unless advised by patient's physician

- Warfarin effect altered with antibiotics and NSAIDs

\section{HIV:}

- Viral load

- CD4 count- >200 cells/mm blood suitable for treatment

- Be aware of common oral manifestations: cervical lymphadenopathy, candidosis, hairy leukoplakia, herpes virus, papilloma virus, aphthous ulcers, Kaposi's sarcoma, and lymphoma. May require biopsy

- Neutropenia

- Bleeding tendency due to risk of thrombocytopenia

- Cross infection risk low but PEP (postexposure prophylaxis) may be required up to 4 weeks if exposure occurs

- IV sedation: benzodiazepine activity may be enhanced with HAART

\section{Malignancy:}

- Malignant spread from organs may manifest in the head and neck region

- Haematological malignancy causes thrombocytopenia(decreased platelets), neutropenia(decreased neutrophils) and anaemia causing increased risk of bleeding and infection

- Patients with bone metastases from carcinomas or with multiple myeloma may be on anti-resorptive medications such as oral or IV bisphosphonates or RANKL inhibitors

\section{Pre-op}

- INR <4 ok for treatment, if >4 refer back to haematology clinic for adjustment

- Dual antiplatelet therapy treat in hospital

\section{Postop}

- Local haemostatic measures

- No NSAIDs

\section{Pre-op}

- Viral Load- <50 viral RNA copies/mm blood, low infectivity suitable for treatment

- CD4 count- >200 cells/mm blood suitable for treatment

- Full blood count, liver profile, coagulation screen

- Antibiotics if neutropenic at risk of infection

Postop

- Antibiotics may be required if neutropenic

- PEP (post-exposure prophylaxis) may be required up to 4 weeks if exposure occurs

\section{Chemotherapy:}

- Risk of bleeding due to thrombocytopenia

- Risk of infection due to neutropenia and immunosuppression

- Anaemia

- Patients may be on high dose steroids

- The above occur usually form the start of therapy until 4 weeks after therapy stops

\section{Pre-op}

- FBC

- Platelets <50 × 109/L may need platelet transfusion

- If severely neutropenic may require antibiotic prophylaxis, liaise with oncologist

Postop

- Haemostatic measures

- Antibiotic therapy

- Tranexamic acid mouthwash (5\%) (though not evidence based may be useful haemostatic adjunctive)

\section{Pre-op}

- Platelets

$<50 \times 109 /$ L platelet transfusion required

- Neutrophils $<1.0 \times 109 / \mathrm{L}$ liaise with oncologist as antibiotic prophylaxis required

- Steroid cover 25 mg IV hydrocortisone if on high dose steroids
7 


\section{Table 1 Management of the medically compromised patient and recent controversies}

\begin{tabular}{|l|l|l}
\hline Medical condition & Recommendations & Reference \\
\hline
\end{tabular}

\section{Continued from page 216}

\section{Radiotherapy:}

- Risk of osteoradionecrosis

- Trismus due to endarteritis can occur 3-6 months after completion of therapy

- Pentoxifylline with vitamin E may be of clinical benefit if ORN occurs

- Patients are at particular risk of osteoradionecrosis when:

- The total radiation dose exceeded $60 \mathrm{~Gy}$

- The dose fraction was large with a high number of fractions.

- There is local trauma from a tooth extraction; periodontal disease or an ill-fitting prosthesis.

- The patient is immunodeficient or malnourished

\section{Bisphosphonates:}

- Risk of ARONJ (anti-resorptive agent induced osteonecrosis of the jaw)

- Anti-resorptive agents include oral and intravenous bisphosphonates and RANKL inhibitors

- An increasing number of patients are being prescribed intravenous bisphosphonates (annually) and RANKL inhibitors (6-monthly) to treat osteoporosis

- Consider risk factors

- Low risk: $<3$ years of oral bisphosphonates, with no other co-morbities

- Medium risk: $>3$ years or oral bisphosphonates and/ or concomitant steroid use and/or concomitant immunosuppressants, intravenous agents prescribed for annual or 6- monthly infusions

- High risk: intravenous agents prescribed previously or currently for metastatic disease

- Type of agent: intravenous agents are more potent than orals but development of ARONJ is dependent on the length of time and dose of the drug prescribed

- Dental factors: periodontal disease, poorly fitting dentures

\section{- Dentoalveolar surgery}

- Local anatomy: tori, maxilla or mandible

- Systemic factors: diabetes, steroids, chemotherapy, immunosuppressants, smoking

\section{- Genetic factors}

\section{Steroids:}

- Risk of Addisonian crisis

- May cause delayed healing
- Extractions should preferably be undertaken up to 3 weeks prior to the start of

treatment, 10 days is considered a minimum period.

- Undertaken in a hospital environment

Pre-op

- Informed consent on risk of osteoradionecrosis

- Antibiotic prophylaxis and $0.2 \%$ chlorhexidine gluconate

Peri-op

- A traumatic extraction use of Luxators and periotomes

Postop

- Postop antibiotics should be prescribed until healing has occurred, may prescribe metronidazole

- $0.2 \%$ chlorhexidine gluconate, qds until healing has occurred

- Hyperbaric oxygen treatment may be recommended before or after treatment

- Avoid wearing dentures until healing has occurred

- Close postop follow up

- Prior to starting anti-resorptive therapy patients should be sent for a thorough dental assessment

If an extraction is required

- Obtain informed consent from the patient

- Atraumatic extractions

- Drug holidays and CTX testing are controversial

- Some centres recommend prophylactic antibiotics

\section{Postop}

- Antibiotics should be prescribed: amoxicillin or metronidazole for 7 days

- $0.2 \%$ chlorhexidine gluconate, qds until healing occurs

Treatment of ARONJ

- Treatment strategies depend on disease staging:

- Stage 0 and 1: analgesics, chlorhexidine mouthwash, antibiotics prescribed for acute episodes of infection

- Stage 2: sequestrectomy together with stage 0 and 1 treatment

- Stage 3: resection of jaw together with stage 0 and 1 treatment

- If stage 2 or 3 treatment is required always consider malignancy as part of differential diagnosis

For surgical procedures with LA or IV sedation.

Pre-op

- 25 mg hydrocortisone IV pre-op or double dose on the day of surgery

General anaesthesia

Pre-op

- 25-50 mg hydrocortisone IV

Postop

- 25-50 mg IM every 6 hours for 24 hours

- May consider antibiotics post-operatively

\section{Sedation:}

- Contra-indicated in:

- Elderly: administer sedation slowly

- Allergy to benzodiazepines

- Pregnancy

- Sickle cell disease: administer 30 mins of oxygen pre- and postop

- Severe COPD (chronic obstructive pulmonary disease)

- Sedate with caution in

- Extremes of age

- Sickle cell disease

- Liver and renal disease

- Myasthenia gravis

- Psychiatric disease 
the patient cannot provide an escort - an increasingly common issue with people living alone - or if the patient has to travel long distances for treatment, this will have a significant impact on their care.

\section{MEDICAL HISTORY}

A consideration of systemic disease is as important in the practice of oral surgery as it is in all other forms of surgery. No procedure should be undertaken until a comprehensive medical history has been ascertained. It is essential that the medical history is:

- Completed at the start of treatment

- Well documented

- Regularly updated.

Whilst there are numerous exhaustive texts on medical conditions in relation to dentistry and minor oral surgery, a great deal can be revealed by clinical observation of the patient together with a few more detailed questions relating to their specific medical conditions. A previous study defined the seven most frequently occurring medical complaints necessitating the referral of patients to hospital for surgical dentistry. Table 1 lists a series of these controversial topics relating to medically complex patients undergoing oral surgery.

If the patient describes a medical condition, some simple questions such as: 'Who treats you?', 'Where and how often are you seen?' will determine the frequency and level of care provided and will often clarify the complexity and severity of their disease.

The fact that a patient is not treated in a specialist hospital unit or admitted does not guarantee that he or she is less severely affected by a particular disease. In general, however, patients who are seen on a regular out-patient basis by consultants are likely to be more severely affected than individuals who are treated on an ad hoc basis by their own family practitioner. In documenting any medical history it is clearly vital to identify the medical practitioners who are treating the patient. They should be informed of any treatment planned, especially where modification of their prescribed treatment is anticipated (particularly anticoagulation therapy prior to surgery).

In order to determine whether or not patients requiring surgery should be treated in the out-patient setting, a riskassessment scale is suggested (Table 2)

Table 2 American Society of Anaesthesiologists' health categorisation

1. Fit and well patients requiring straightforward surgery who are expected to make a complete and uneventful recovery

2. Patients with a medical condition that may complicate the delivery of care but is unlikely to affect outcome (eg hepatitis, past history of endocarditis, steroids, epilepsy, mental handicap)

3. Patients with medical condition(s), or past surgery, which may additionally compromise outcome (complicated surgery, uncontrolled diabetes, coagulopathies, immunosuppression)

4. Patients in whom the complications of surgery may be severe with marked local or systemic complications (inherited clotting disorders, uncontrolled local or systemic disease) and/or require contemporaneous specialised medical therapy (severe immunosuppression, haemophilia).

5/6. Not relevant to oral surgery

based on that developed for anaesthetic risk-assessment by the American Society of Anaesthesiologists. In the proposed scale there are four relevant groups of patient.

It is recommended that patients in groups one and two may be routinely treated in the out-patient or practice setting. Patients in group three may be treated in the practice setting at the discretion of the individual operator, provided that appropriate precautions have been taken. Patients in group four, irrespective of the nature of the surgery required, should always be treated in the hospital environment.

\section{SPECIFIC CONDITIONS THAT MAY SIGNIFICANTLY ALTER SURGICAL OUTCOMES}

\section{Smoking}

Dental patients who smoke need access to local services to help them stop smoking. A dentist who recognises a patient as a smoker has a duty to inform the patient of the options available to them. Dentists can help their patients to stop smoking by recognising oral signs of tobacco use, informing patients of these and asking patients whether they wish to stop. They can then refer those patients who wish to stop smoking to smoking cessation services. Not all smokers are ready to quit smoking; some have not considered quitting. Others may be considering stopping but may not be sure how to take the next steps. By enquiring and providing advice, members of the dental team can help to encourage patients from pre-contemplation through contemplation towards action. ${ }^{2}$ In this regard, the 'four As' model consists of:

- Asking about smoking and the desire to stop

- Advising of the value of quitting

- Assisting the patient to stop through access to appropriate support - Arranging follow up support. ${ }^{3}$

\section{Obesity}

Obesity is considered to be an ongoing epidemic in the western world with an estimated $31 \%$ of the adult population classified as obese. The World Health Organisation (WHO) classifies obesity as a chronic disease. Affected individuals are at increased risk of hypertension, heart disease, diabetes and other illnesses. There are also important social implications within dentistry and, particularly in the provision of conscious sedation, obesity can be a potential complicating factor. ${ }^{21}$ The dentist's role in providing dietary advice with the aim in reducing dental caries would also be appropriate for obesity reduction. Evidence linking a reduction of obesity and related diseases and a higher oxidative diet (fruit, vegetables, nuts and less than two units of alcohol) is increasing. Exciting developments have linked periodontitis and other focal inflammation as indicators for oxidative stress and other related systemic disorders including ischaemic heart disease, diabetes, stroke and obesity. ${ }^{6}$

Thus, in future, the dentist may be expected to identify obese patients who may be at risk of ischemic heart disease, stroke or diabetes and may play a significant role in disease prevention.

\section{Cardiovascular disease}

Cardiovascular disease is common in Western populations and every dental practitioner will encounter patients with cardiac disease at some stage. The most common types are ischaemic heart disease (IHD), hypertension, cardiac failure, cardiac dysrhythmias and valvular heart disease. ${ }^{6}$

Ischaemia of the heart muscle occurs when the oxygen supply does not meet the 
myocardial demand. This is usually caused by coronary artery disease where atheromatous plaques in the coronary arteries obstruct blood flow to the myocardium. Myocardial ischaemia is responsible for stable angina (cardiac chest pain on exertion that is relieved by rest) and the spectrum of acute coronary syndromes from unstable angina (angina of recent onset, increasing frequency or severity or occurring at rest) and myocardial infarction. It is often useful to ask a patient who suffers from angina to use their glyceryl trinitrate (GTN) spray prior to dental treatment. ${ }^{4}$

Hypertension is persistently raised blood pressure due to increased arteriolar resistance. In the general population, 90\% of cases relate to essential hypertension with no predisposing cause. Occasionally hypertension is secondary to another condition, usually renal disease or diabetes mellitus. A sustained BP (blood pressure) $>140 / 90 \mathrm{mmHg}$ is considered hypertensive. ${ }^{5}$

Cardiac failure occurs when the heart is unable to maintain sufficient cardiac output to meet the demands of the body despite venous pressures being normal. The most common causes are ischaemic heart disease, hypertension and chronic obstructive pulmonary disease (COPD).

Oral surgery treatment should be planned to minimise the risk of precipitating an acute cardiac event. Patients should be treated in the late morning or early afternoon when levels of endogenous circulating catecholamines are lower and appointments should be kept short. Measures should be taken to minimise anxiety and treatment must be pain-free with excellent local analgesia. Treatment under intravenous sedation may be beneficial but should be performed in the hospital setting. Intravenous sedation should, however, be used with extreme caution and may be contraindicated in patients with corpulmonale (right heart failure secondary to pulmonary disease) due to its respiratory suppressant effects.

Patients with severely elevated blood pressure (>160/100 $\mathrm{mmHg}$ ) should be referred to a medical practitioner for investigation and control of their blood pressure prior to elective surgery. Use of intravenous sedation with midazolam is advocated in hypertension where it has been shown to lower blood pressure during oral surgery procedures. ${ }^{4}$ Blood pressure should be recorded throughout treatment in patients with uncontrolled hypertension and treatment ceased (or patient refered to their GMP) if significant increases occur.

Local anaesthetic agents containing epinephrine are not contraindicated but the dose should be limited to two or three cartridges and an aspirating syringe used. Patients taking $\beta$-blockers in particular may experience an increase in systolic blood pressure with local anaesthetic containing epinephrine. Use of non-steroidal anti-inflammatory drugs (NSAIDs) is best avoided in patients taking angiotensinconverting enzyme (ACE) inhibitors (eg ramipril) where they may cause a deterioration in renal function. Patients with cardiac failure often become breathless on lying flat (orthopnoea) due to gravitational redistribution of blood to the pulmonary circulation and should therefore not be placed fully supine in the dental chair. Many antihypertensive medications can cause postural hypotension, which may lead to transient loss of consciousness if raised rapidly from a lying to sitting position. Symptoms of acute cardiac events include chest pain, dyspnoea, palpitations and syncope, which can lead to cardiac arrest; all require urgent management and medical intervention if they occur in the dental setting.

Antibiotic prophylaxis (Table 1) for prosthetic and diseased heart valves and congenital heart defects should no longer be given. ${ }^{5}$ The risk of an adverse outcome following administration of prophylactic antibiotics exceeds the likelihood of infective endocarditis being prevented.

\section{Respiratory disease}

Asthma, chronic obstructive pulmonary disease (COPD) and respiratory tract infections are commonly encountered in dental practice. Asthma is a chronic inflammatory condition of the lung airways. It is characterised by reversible airflow limitation, hyper-responsiveness of the airways to a range of stimuli and bronchial inflammation. By contrast, COPD is characterised by poorly reversible airflow limitation that is progressive and associated with persistent lung inflammation. COPD is almost exclusively caused by smoking and the term encompasses both chronic bronchitis and emphysema.
Elective oral surgery procedures should be deferred in patients with acute exacerbations of respiratory disease. Patients with asthma should be asked to bring their inhalers when attending for treatment and use them prior to treatment. Aspirin and NSAIDs may induce an asthma attack and should be avoided in sensitive individuals. Intravenous sedation may be beneficial in patients whose asthma is exacerbated by anxiety but should be performed with caution in the hospital environment. General anaesthesia and intravenous sedation are best avoided in patients with COPD. Use of systemic corticosteroids exceeding $15 \mathrm{mg}$ daily of prednisolone or equivalent may require steroid cover to prevent hypotensive collapse during treatment. Aspirin and NSAIDs are best avoided in patients taking long-term corticosteroids due to the increased risk of peptic ulceration.

Leukotriene-receptor antagonists (eg montelukast) used in the treatment of asthma may impair liver function and increase bleeding tendency.

Theophylline levels can be potentiated by drugs such as epinephrine and erythromycin and precipitate cardiac dysrhythmias. Patients with COPD are best treated in the upright position to avoid exacerbating breathlessness.

\section{Disorders of haemostasis}

The most common disorders of haemostasis are anticoagulant therapy, hepatic and renal disease. Congenital bleeding disorders and bone marrow failure are rare but important causes of excessive bleeding.

Warfarin is prescribed to patients with atrial fibrillation to prevent strokes, or to patients who have a history of recurrent deep vein thrombosis (DVT) or pulmonary embolism (PE). Patients taking warfarin should have their International Normalised Ratio (INR) checked on the day of surgery, or if that is not possible on the day before. The therapeutic range for INR is usually 2-3 for DVT and up to 3.5 for prosthetic heart valves. An INR of less than four is acceptable for routine forceps extraction of up to three teeth or a single surgical procedure. If the INR exceeds four, treatment should be deferred and the patient's anticoagulation clinic contacted as there is no therapeutic indication to have an INR above four. Adjustments to warfarin dosage should only be made by physicians, 
as life threatening thrombosis may result from stopping anticoagulant treatment. The routine use of local measures to control haemostasis following surgical procedures is advised in all warfarinised patients. These include suturing and packing extraction sockets with a resorbable haemostatic agent such as SurgicelR. The anticoagulant effect of warfarin can be potentiated by NSAIDs and numerous antibacterials including amoxicillin, erythromycin and metronidazole. These drugs should be avoided in warfarinised patients. ${ }^{8,22}$

New oral anticoagulants include dabigatran, a direct thrombin inhibitor and rivaroxaban, a factor Xa inhibitor. These drugs do not require routine coagulation monitoring using the INR. Most dental treatment - such as the extraction of a small number of teeth - would be comparable to treating a patient on warfarin with an INR less than or equal to four, relying on local measures to obtain haemostasis. NSAIDs should still be avoided due to their antiplatelet activity and paracetamol is the analgesic of choice. ${ }^{9}$

Aspirin disrupts platelet aggregation and is used as prophylaxis against cardiac events and stroke in patients with cardiovascular disease. Other antiplatelet drugs used in the prevention of occlusive vascular events include clopidogrel, dipyridamole and glycoprotein IIb/IIIa inhibitors such as abciximab. Heparin is administered subcutaneously or intravenously in acute thrombotic states such as DVT and PE. Antiplatelet drugs rarely cause problematic bleeding following minor surgical procedures and do not need to be stopped pre-operatively. Use of local measures to aid haemostasis is, however, advisable.

Parenchymal liver disease and obstructive jaundice can both cause bleeding tendency. Pre-operative haematological investigation is indicated to check the full blood count (FBC), INR and activated partial thromboplastic time (APTT). A physician should be consulted prior to surgical treatment if significant abnormalities are detected. In the presence of severe bleeding tendency, parenteral administration of vitamin K1 may be indicated for several days pre-operatively or transfusion of fresh frozen plasma may be required.

A number of medical conditions can cause thrombocytopenia (low platelets).
These include chronic liver disease, renal failure, bone marrow failure, HIV, idiopathic thrombocytopenic purpura (ITP), leukaemia and systemic lupus erythematosus (SLE). A full blood count should be requested to check platelet levels prior to surgery. A platelet level $>50 \times 109 / \mathrm{L}$ is considered adequate in patients undergoing dentoalveolar surgery although local measures to control haemostasis should be used routinely. If platelets are below this level a haematologist should be consulted, as pre-operative platelet transfusion is usually required.

Congenital bleeding disorders such as haemophilias A, B and C, Von Willebrand's disease and individual clotting factor deficiencies always require liaison with the patient's haematologist and are best managed in the hospital setting.

All patients with disorders of haemostasis should be treated in the morning and early in the week. Extraction of multiple teeth may require treatment in stages over multiple visits. Surgery should be atraumatic, avoiding inferior dental (ID) blocks and raising flaps where possible. Local measures for haemostasis should be used routinely.

Anaemia is a haemoglobin ( $\mathrm{Hb}$ ) level below normal for the age and sex of the individual. Haemoglobin levels $<11.5 \mathrm{~g} / \mathrm{dl}$ in adult females and $<13.0 \mathrm{~g} / \mathrm{dl}$ in adult males are considered criteria of anaemia. Anaemia is not a disease in itself but rather a feature of many types of disease. In developed countries the most common cause is iron deficiency resulting from chronic blood loss, usually excessive menstrual blood loss in women. Elective surgery under general anaesthetic (GA) in patients with $\mathrm{Hb}<10 \mathrm{~g} / \mathrm{dl}$ should be deferred until the anaemia has been corrected. Conscious sedation should be used with caution and supplemental oxygen administered to prevent hypoxia. It should be borne in mind that $70 \%$ of menstruating women are iron deficient; however, if an older male patient presents with signs of anaemia, occult blood loss due to bowel cancer should be excluded.

Sickle cell anaemia is a genetic abnormality of the globin chain resulting in abnormally shaped erythrocytes that readily infarct small blood vessels. Sickle cell screening must be performed for all Africans and Afro-Caribbeans prior to general anaesthesia. Intravenous sedation with benzodiazepines is best avoided in patients with sickle cell disease. Intravenous sedation is, however, preferable to general anaesthesia as it is associated with lower risk and is more cost effective and if required should be performed in hospital with pre- and peri-operative oxygenation. Aspirin is best avoided as it may cause acidosis and precipitate a crisis. Prophylactic antibiotics may be considered for surgical procedures and infections must be treated vigorously. Patients who are carriers (sickle cell trait) require no special precautions other than a general screening for coincidental anaemia.

\section{LIVER DISEASE}

The most common causes of parenchymal liver disease are alcohol-induced cirrhosis and viral hepatitis. Other less common causes include auto-immune or druginduced hepatitis and primary biliary cirrhosis. Occasionally, hepatocellular disease may be a result of congenital hyperbilirubinaemia or extrahepatic biliary obstruction (eg by gallstones).

The main significance to dentists of liver disease is impaired synthesis of clotting factors, resulting in bleeding tendencies and impaired drug metabolism. Erythromycin and tetracyclines are hepatotoxic and should be avoided. Clindamycin, metronidazole and paracetamol may be used in reduced doses and penicillins are safe at normal doses. Aspirin and NSAIDs should be avoided due to risk of haemorrhage in patients with portal hypertension and oesophageal varices. Patients with chronic liver disease may have increased susceptibility to infection and any infections should be managed vigorously. Metabolism of benzodiazepines is impaired and IV sedation should be performed with caution, in a specialist setting. Viral hepatitis poses an infection risk and rigorous cross-infection control measures are essential to prevent disease transmission.

\section{Renal disease}

Of greatest relevance to dental practitioners is chronic kidney disease (CKD), also known as chronic renal failure. Common causes of CKD include diabetes mellitus, hypertension, renovascular disease, pyelonephritis and polycystic kidney 
disease. The effects of CKD include bleeding tendencies due to thrombocytopenia, increased susceptibility to infections and enhanced activity of drugs excreted by the kidney.

Pre-operative haematological investigation should include a full blood count to check platelet levels and white cell count, urea and electrolytes and estimated glomerular filtration rate (GFR) to assess the degree of renal impairment.

Care must be taken to ensure adequate haemostasis and prophylactic antibacterials may be indicated for surgical procedures. Amoxicillin, erythromycin, metronidazole, paracetamol and codeine are relatively safe and adjustment to dosage is only required if renal failure is severe. Aspirin and NSAIDs are nephrotoxic and should be avoided.

Patients with end-stage renal failure require haemodialysis or renal transplant. Treatment for patients on dialysis should be booked on a day following dialysis when the beneficial effects are maximal and the effects of heparin have worn off. Intravenous sedation with midazolam may be used but cannulation of the veins of the forearms and arteriovenous shunts used for dialysis must be avoided.

Patients who have received a renal transplant will be taking immunosuppressant therapy. They usually require prophylactic antibacterials for oral surgical procedures and management should be planned following consultation with the renal physician. Those taking long-term high-dose corticosteroids require steroid cover.

\section{Diabetes}

Diabetes mellitus refers to metabolic disorders characterised by chronic hyperglycaemia resulting from insulin deficiency, insulin resistance or both. It is usually primary but may also be secondary to chronic pancreatitis, Cushing's syndrome or drug-induced by thiazide diuretics or corticosteroids.

In type I (insulin dependent) diabetes there is an absolute lack of insulin and insulin therapy is always required. In type II (non-insulin dependent) diabetes there is insulin deficiency and peripheral resistance to its actions. Insulin therapy may still be indicated if glycaemic control is not adequately achieved by diet and oral hypoglycaemics. ${ }^{5}$
Diabetic patients should be given appointments early in the morning to ensure treatment does not interfere with mealtimes or insulin regimes. Blood glucose levels should be checked using blood glucose test strips ('BM stix') prior to surgery. Treatment can be delivered safely if blood glucose is in the range 5.0-15.0 mmol/L. Oral glucose should be given pre-operatively to patients with a glucose level $<5.0 \mathrm{mmol} / \mathrm{L}$ and elective surgery in poorly controlled diabetics should be deferred until glycaemic control is improved. Diabetic patients have increased susceptibility to infection and vigorous treatment of active infection is essential although prophylactic antibacterials following minor surgical procedures are not indicated. All dentists must be familiar with the signs and symptoms of a hypoglycaemic attack, which should be managed as a medical emergency if it occurs in the dental setting.

Obese patients with diabetes mellitus and ischaemic heart disease are likely to be suffering from metabolic syndrome.

\section{Epilepsy}

Surgical procedures in poorly controlled epileptics are best performed in the hospital setting. Treatment under intravenous sedation with midazolam is advocated due to the anticonvulsant effect of benzodiazepines. Epileptic seizures and status epilepticus may occur in the dental setting and require emergency management, protection of the airway being of paramount concern.

\section{Immunodeficiencies and HIV}

These are usually the result of HIV infection, bone marrow disease such as leukaemia and aplastic anaemia and immunosuppressant therapy. Immunosuppressive drugs are used to prevent organ rejection in transplant recipients, treat autoimmune disorders, connective tissue diseases and some neoplasms, particularly the haematological malignancies.

HIV positive patients with a CD4 count (a glycoprotein expressed on the surface of T helper cells, monocytes, macrophages) below 200 and all other immunosuppressed patients should have a full blood count prior to treatment. If there is significant leukopenia or neutropenia, prophylactic antibacterials are indicated for surgical procedures. Orofacial infections should be treated vigorously with antimicrobials. Thrombocytopenia is common in immunosuppressed individuals causing bleeding tendency and those on long-term corticosteroids require steroid cover for surgical procedures.

\section{Malignant disease}

Both radiotherapy and chemotherapy ${ }^{10}$ are discussed in Table 1.

\section{Medications}

See Table 1 for information on sedation, chemotherapy ${ }^{10}$ and bisphosphonates. ${ }^{11-19}$

\section{Allergies}

Increasingly, patients report allergies to commonly-used agents in dentistry. The most common allergy relevant for dental patients is natural latex allergy. Approximately $1 \%$ of the population has a latex allergy. ${ }^{23}$ Anyone who is in frequent contact with latex-containing products is at risk of developing a latex allergy. The populations at highest risk are medical and dental professionals, workers in latex companies and patients with spina bifida. Other individuals at risk include patients:

- With a history of asthma or other allergies, especially to bananas, avocados, tropical fruits, or chestnuts

- With a history of multiple intraabdominal or genito-urinary surgeries

- Who require frequent intermittent urinary catheterisation.

The second most common allergy is to the penicillins with 2-3\% of the population affected. Approximately 400 people die in the USA each year as a result of penicillin allergy. $^{23}$

Allergy to local anaesthetic (LA) agents is extremely rare and has previously been attributed to the LA cartridge, which was manufactured using latex elements. This is no longer the case as most dental LA manufacturers use latex-free cartridge bungs. Patient collapse during LA administration is most likely to be due to a vasovagal attack (faint).

\section{Creutzfeldt-Jakob disease (CJD)}

CJD is a rare but significant entity due to prion disease. Prions are thought to be the smallest form of infectious particles and are not destroyed by conventional sterilising techniques. If patients are recognised as 
being at heightened risk of infection with any form of CJD, instruments used on them are already subject to special precautions against onward infection, being quarantined and, if necessary, destroyed. Patients at risk of CJD include those with close family members with a diagnosis of CJD and patients who have previously undergone dural or corneal grafts and human growth factor therapy. A single dental procedure on an infective patient is estimated to be about 1,000,000,000 times less likely to transmit variant CJD than - say - a tonsillectomy. The latter, in turn, would be much less likely to do so than a procedure involving the central nervous system or the back of the eye. ${ }^{24}$

\section{INVESTIGATIONS}

Every effort should be made at the time of the operation to minimise or avoid complications and side effects of the operative procedures. Thus familiarity with the patient's medical and social histories is essential. Preoperative investigations may include blood tests for the indications above, radiographs, scans, study models and occasionally liaison with other specialists to maximise treatment benefit.

\section{Radiological assessment}

The purpose of a careful radiological evaluation is to complement the clinical examination by providing additional information about the tooth to be extracted, the related teeth and anatomical features, and the surrounding bone. This is necessary in order to make a sound decision about the proposed surgical procedure, the most appropriate location for this to take place, and to highlight aspects of management that may require specific discussion with the patient.

Prior to the age of 13 , radiographic examination is not normally indicated for the assessment of third molars and films taken from the age of 20 are most useful in assessing the likelihood of eruption. When more than one third molar requires assessment, the radiographic examination of choice is a panoramic radiograph as the radiation dose of a panoramic radiograph is lower than from four periapical views and the diagnostic yield higher. Doses from panoramic radiography can be further limited by using field size limitation to prevent exposing areas not required in the field of view. Periapical or oblique lateral radiographs may be taken as an alternative. All radiographs should be of a diagnostically acceptable standard and optimised for radiation dose in accordance with IR(ME)R (2000) regulations. ${ }^{25}$

The effectiveness of cone beam CT scans providing coronal and sagittal reformatting in establishing the three dimensional relationship of the orofacial region has become an increasing popular modality. CBCT permits localisation of the inferior alveolar nerve (IAN) canal in relation to the mandibular third molar (M3M) and the inferior dental canal (IDC). Detection of an intra-radicular path; determination of the distance between the tooth and IAN canal, and root angulation are clearly identifiable using this additional radiography once a dental panoramic tomograph (DPT) has established that the M3M is close to, crossing or superimposed on to the IDC, thus minimising IAN injury on removal of the high risk tooth. If the IAN is entrapped within the substance of the tooth, coronectomy or monitoring may be indicated. Knowing the IDC position relative to the third molar may decrease the frequency or severity of IAN injury.

CBCT may also be useful for complex pathology or severely ectopic non-molar teeth; however, the increased radiation dose of CBCT must be considered when ordering these images. A DPT has a dose in the range of 16-30 $\mu$ Svs compared with a conventional medical CT of 300$600 \mu$ Svs. Cone beam CT scan doses range from $60-180 \mu$ Svs.

Overall, the combined clinical and radiographic assessment for oral surgery is to enable the clinician to make a diagnosis that may be concordant with indications for removal of the tooth leading to surgical planning.

Radiographs should not only demonstrate the area of interest but be interpretable and clearly identifiable (dated and named). Whilst intra-oral radiographs are perfectly adequate for most types of surgery, DPTs (despite their limitations for the visualisation of crown pathology, anterior teeth and recurrent caries) are increasingly favoured in surgical practice as they provide a useful way of screening the jaws for pathological changes. Radiographs will demonstrate not only the intrinsic factors that can make an extraction or other procedure difficult (eg angulation, root morphology, caries and ankylosis) but also the relationship of the area of surgical interest to vital anatomical structures, such as the maxillary sinus or inferior alveolar neurovascular bundle.

Finally, the surgeon should plan treatment at a time and place that is convenient, well-equipped and where the facilities allow for dealing with any complication. Efficiency and effectiveness are the hallmarks of a good surgeon and these should improve with time and experience.

1. Medical Education England. Review of oral surgery services and training. Online review available at http://www.mee.nhs.uk/our_work/work_priorities/ review_of_oral_surgery.aspx (accessed July 2013).

2. Monaghan N. What is the role of dentists in smoking cessation? Br Dent J 2002; 193: 611-612.

3. Dent TH. Predicting the risk of coronary heart disease I. The use of conventional risk markers. Atherosclerosis 2010; 213: 345-351.

4. Woolcombe S, Koshel S, Bryant C, Rood P. Use of intravenous sedation in the management of patients with high blood pressure. Oral Surgery 2009; 2: 116-125.

5. National Institute for Health and Care Excellence. Antimicrobial prophylaxis against infective endocarditis. London: NICE, 2008.

6. Bollino D, Marigo L. Oral inflammatory process and general health. Part 1: The focal infection and the oral inflammatory lesion. Eur Rev Med Pharmacol Sc 2010; 14: 1085-1095.

7. Scully C. Medical problems in dentistry. 6th ed. London: Churchill Livingstone, 2010.

8. US Food and Drug Administration. Algorithm for warfarinised patients in dentistry. Philadelphia: FDA, 2007.

9. Griffiths M, Scully C. New anticoagulants. Br Dent J 2012; 213: 96.

10. The Royal College of Surgeons of England, The British Society for Disability and Oral Health. The oral management of oncology patients requiring radiotherapy, chemotherapy and bone marrow transplantation, clinical guidelines. London: RCS, 2012. Online guideline available at http://www.rcseng.ac.uk/fds/publicationsclinical-guidelines/clinical_guidelines/documents/ clinical-guidelines-for-the-oral-management-ofoncology-patients-requiring-radiotherapy-chemotherapy-and-or-bone-marrow-transplantation (accessed July 2013)

11. Marx R E. Oral and intravenous bisphosphonateinduced osteonecrosis of the jaws. 2nd ed. London: Quintessence Publishing Co, Inc., 2011.

12. Ruggiero $S L$, Dodson $T B$, Assael $L A$, Landesberg $R$, Marx R E, Mehrotra B. American Association of Oral and Maxillofacial Surgeons' position paper on bisphosphonates related osteonecrosis of the jaws 2009 update. J Oral and Maxillofac Surg 2009; 67: 2-12.

13. Ruggiero S L. Bisphosphonate-related osteonecrosis of the jaw: an overview. Ann N Y Acad Sci 2010; 1218: 38-46.

14. McLeod N M, Bater M C, Brennan P A. Management of patients at risk of osteoradionecrosis: results of a survey of dentists and oral and maxillofacial surgery units in the UK, and suggestions for best practice. Br J Oral Maxillofac Surg 2010; 48: 301-304.

15. Scottish Dental Clinical Effectiveness Programme Oral health management of patients prescribed bisphosphonates. Edinburgh: SDCEP, 2011.

16. Malden N, Beltes $\mathrm{C}$, Lopes V. Dental extractions and bisphosphonates: the assessment, consent and management, a proposed algorithm. Br Dent J 2009; 206: 93-98.

17. Sarasquete M E, Gonzalez M, San Miguel J F, 
Garcia-Sanz R. Bisphosphonaterelated osteonecrosis: genetic and acquired risk factors. Oral Dis 2009; 15: 382-387.

18. Suzuki J, Lee C. ADI 2012 White Paper on antiresorptive therapy and osteonecrosis of the jaws. Association of Dental Implantology UK, 2012. Online article available at http://www.lciad. co.uk/uploads/pdf/ADI_2012_White_Paper_on_ ARONJ___Suzuki_Et_Lee.pdf (accessed July 2013).

19. Mcleod N M H, Patel V, Kusanale A, Rogers S N, Brennan P A. Bisphosphonate osteonecrosis of the jaw: a literature review of UK policies versus international policies on the management of bisphosphonate osteonecrosis of the jaw. Br J Oral and Maxillofac Surg 2011: 49: 335-342.

20. North West Medicines Information Centre. Surgical management of the primary care dental patient on antiplatelet medication. Liverpool: North West Medicines Information Centre, 2009.

21. Reilly D, Boyle C A, Craig D C. Obesity and dentistry: a growing problem. Br Dent J 2009; 207: 171-175.

22. NHS. Management of routine exodontia in warfarinised patients undergoing surgical procedures in primary care. Online information available at http://www.nyypct.nhs.uk/Advicelnformation/ ReferralToolkit/docs/Management of routine exodontia.pdf (accessed July 2013)

23. Better Medicine. Allergy facts. Online information available at http://www.bettermedicine.com/topic/ allergies (accessed July 2013).

24. Department of Health. Risk assessment for VCJD in dentistry. London: $\mathrm{DH}, 2003$. Online article available at $\mathrm{http}: / /$ webarchive.nationalarchives. gov.uk/20130107105354/http://www.dh.gov. uk/en/Publicationsandstatistics/Publications/ PublicationsPolicyAndGuidance/DH_4084662 (accessed July 2013).

25. The ionising radiation (medical exposure) regulations 2000. Online regulations available at www. dh.gov.uk/prod_consum_dh/groups/dh_digitalassets/@dh/@en/documents/digitalasset/dh_064707. pdf (accessed July 2013).

\section{Erratum}

\section{CPD questions (BDJ 2013; 215: 190)}

CPD Article 1 - The use of diffuse laser photonic energy and indocyanine green photosensitiser as an adjunct to periodontal therapy

Question 4 should have read as follows:

The use of a non-ablation, low-level laser wavelength to initiate photodynamic antimicrobial chemotherapy in a suitably administered photosensitiser may be seen to have the following advantages over 'conventional' laser use, except for the following:

This amendment was incorporated on the BDA CPD Hub website as soon as the incorrect wording of this question was noticed.

We apologise for any confusion and inconvenience caused. 